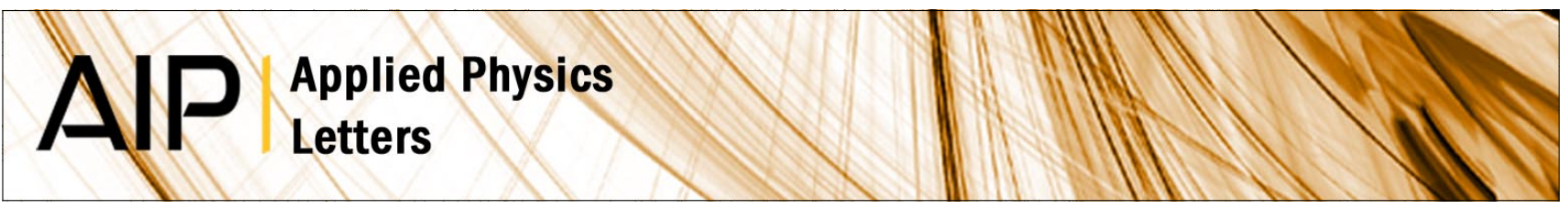

\title{
Field enhanced bulk conductivity of acceptor-doped BaTi1xCaxO3x ceramics
}

Nahum Masó, Marta Prades, Héctor Beltrán, Eloisa Cordoncillo, Derek C. Sinclair et al.

Citation: Appl. Phys. Lett. 97, 062907 (2010); doi: 10.1063/1.3476355

View online: http://dx.doi.org/10.1063/1.3476355

View Table of Contents: http://apl.aip.org/resource/1/APPLAB/v97/i6

Published by the American Institute of Physics.

\section{Related Articles}

Charge dynamics in KH2PO4 systematically modified by proton irradiation

J. Appl. Phys. 110, 044101 (2011)

Effect of grain sizes and shapes on phonon thermal conductivity of bulk thermoelectric materials J. Appl. Phys. 110, 024312 (2011)

Improved carrier concentration control in Zn-doped Ca5Al2Sb6

J. Appl. Phys. 110, 013721 (2011)

High-temperature transport properties of Ca0.98RE0.02MnO3 (RE $=\mathrm{Sm}, \mathrm{Gd}$, and Dy)

Appl. Phys. Lett. 98, 214101 (2011)

Nonlinear transport properties and Joule heating effect in charge ordered LuFe2O4

Appl. Phys. Lett. 98, 102102 (2011)

\section{Additional information on Appl. Phys. Lett.}

Journal Homepage: http://apl.aip.org/

Journal Information: http://apl.aip.org/about/about_the_journal

Top downloads: http://apl.aip.org/features/most_downloaded

Information for Authors: http://apl.aip.org/authors

\section{ADVERTISEMENT}

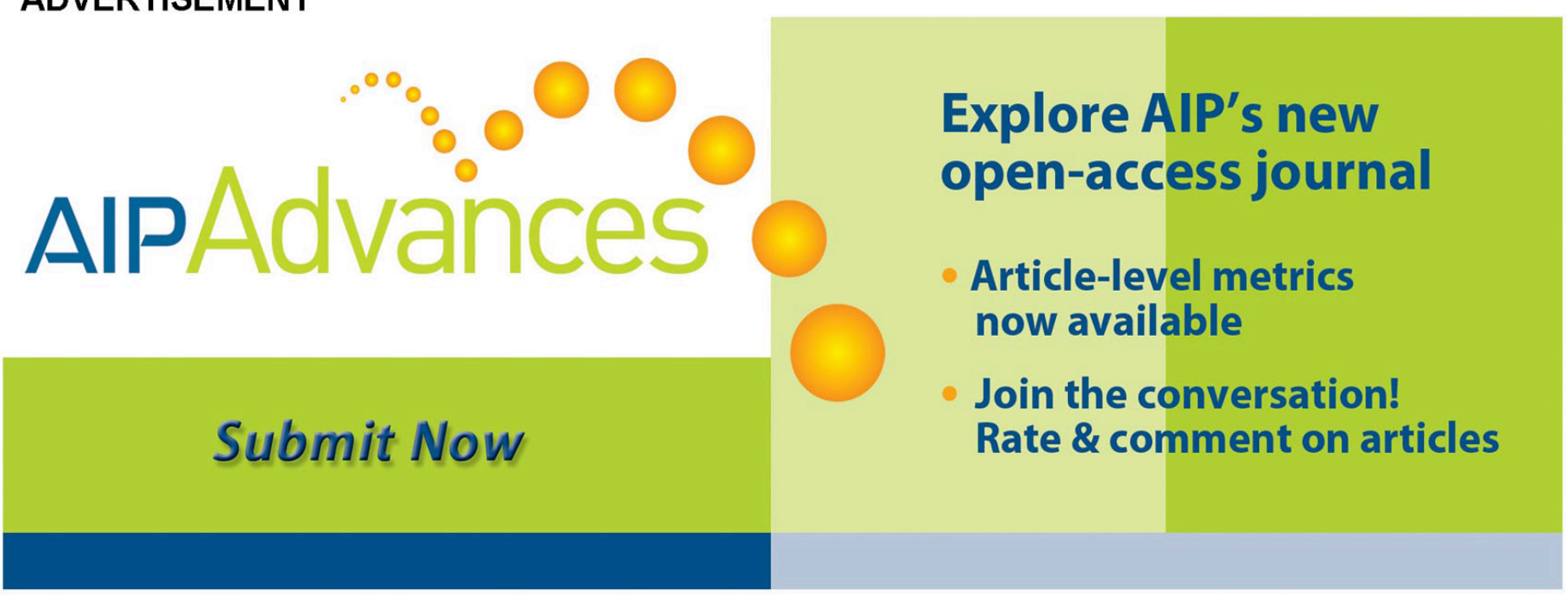




\title{
Field enhanced bulk conductivity of acceptor-doped $\mathrm{BaTi}_{1-\mathrm{x}} \mathrm{Ca}_{\mathrm{x}} \mathrm{O}_{3-\mathrm{x}}$ ceramics
}

\author{
Nahum Masó, ${ }^{1}$ Marta Prades, ${ }^{2}$ Héctor Beltrán, ${ }^{2}$ Eloisa Cordoncillo, ${ }^{2}$ Derek C. Sinclair, ${ }^{1}$ \\ and Anthony R. West ${ }^{1, \text { a }}$ \\ ${ }_{1}^{1}$ Department of Engineering Materials, The University of Sheffield, Sheffield S1 3JD, United Kingdom \\ ${ }^{2}$ Departamento de Química Inorgánica y Orgánica, Universidad Jaume I, 12071 Castellón, Spain
}

(Received 13 April 2010; accepted 16 July 2010; published online 10 August 2010)

\begin{abstract}
The electrical properties of Ca-doped $\mathrm{BaTiO}_{3}$ are very different when $\mathrm{Ca}$ substitutes onto $\mathrm{Ba}$ or $\mathrm{Ti}$ sites. The $p$-type semiconductivity of Ti-substituted ceramics increases reversibly by one to two orders of magnitude under a $d c$-bias voltage of $\leq 100 \mathrm{~V} \mathrm{~cm}^{-1}$, whereas Ba-substituted ceramics show little sensitivity to a $d c$ bias. This increase in $\mathrm{BaTi}_{1-\mathrm{x}} \mathrm{Ca}_{\mathrm{x}} \mathrm{O}_{3-\mathrm{x}}$, studied over the temperature range $150-600{ }^{\circ} \mathrm{C}$, is independent of electrode material and atmosphere and is attributed to ionization of underbonded $\mathrm{O}^{2-}$ ions adjacent to acceptor-doped $\mathrm{Ca}^{2+}$ ions. () 2010 American Institute of Physics. [doi:10.1063/1.3476355]
\end{abstract}

The electrical properties of $\mathrm{BaTiO}_{3}$ (BT) ceramics are sensitive to dopants and in particular, whether they are acceptors (ions with lower valence than the host ion), donors (higher valence), or isovalent (same valence). Acceptor dopants, e.g., $\mathrm{Ca}$ in place of $\mathrm{Ti}$, are important for increasing the voltage stability of BT-based ceramics since associated charge compensation, involving creation of oxygen vacancies, acts to reduce loss of oxygen during high temperature processing. ${ }^{1}$ Although acceptor-doped materials are less sensitive to reduction, which is important in firing multilayer ceramic capacitors (MLCCs) in reducing atmospheres with base metal electrodes (BME) such as $\mathrm{Ni}^{2}{ }^{2}$ this advantage may be at least partially offset by the presence of mobile oxygen vacancies which are thought to be important in electrical degradation.

$\mathrm{Ca}$ is an unusual dopant for BT since, as also for intermediate-sized rare earths, it can substitute for either Ba (A-site) or Ti (B-site) and the resulting electrical properties differ greatly. For A-site substitution (referred to as BCT), the ferroelectric Curie temperature $T_{c}$, grain conductivity, polarization-electric field (P-E) loops and strain-E (e-E) curves change little with $\mathrm{Ca}$ content. With B-site substitution (BTC), dramatic changes are observed: ${ }^{3,4} \mathrm{~T}_{\mathrm{c}}$ decreases by $\sim 22{ }^{\circ} \mathrm{C} /$ at. $\%$ and the grain conductivity increases by several orders of magnitude; reversible ferroelectric domain switching with remanent polarization, $\mathrm{P}_{\mathrm{r}} \sim 0 \mu \mathrm{C} / \mathrm{cm}^{2}$ (double P-E hysteresis loops) and significant nonlinear re- coverable electrostrain of $\sim 0.04 \%$ at $\sim 11 \mathrm{kV} / \mathrm{cm}$ occur at room temperature. ${ }^{3}$ The different behavior of BTC has been attributed to mobility of oxygen vacancies created by acceptor doping and a tendency for the defect dipole to align with the crystal symmetry. ${ }^{5}$

Here, we report another major difference in electrical properties for the two substitution mechanisms: Ti-site substitution leads to field-enhanced bulk conductivity whereas Ba-site substitution shows little dependence of conductivity on $d c$ bias. This effect is not associated primarily with mobility of oxygen vacancies but instead, is attributed to the ionization of underbonded oxide ions adjacent to $\mathrm{Ca}$ acceptor dopants.

Two series of compositions were made with 1 and 3\% substitution of Ca onto A and B sites, Table I. Samples were prepared using sol-gel methods ${ }^{6,7}$ with high purity chemicals. $\mathrm{Ca}\left(\mathrm{CH}_{3} \mathrm{COO}\right)_{2} \cdot \mathrm{H}_{2} \mathrm{O}$ [99\%, Sigma-Aldrich] was used as $\mathrm{Ca}$ precursor. Homogeneous gel products were gradually heated to $1400{ }^{\circ} \mathrm{C}$ for $2 \mathrm{~h}$, pressed into pellets, fired in air at either $1400{ }^{\circ} \mathrm{C}$ (A-site) or $1500{ }^{\circ} \mathrm{C}$ (B-site) for $12-24 \mathrm{~h}$ and cooled slowly to room temperature by switching off the furnace. Completeness of reaction was confirmed by x-ray powder diffraction and lattice parameters obtained by leastsquares refinement of reflections in the range $15^{\circ} \leq 2 \theta$ $\leq 90^{\circ}$ using a Stoe diffractometer, $\mathrm{Cu} \mathrm{K} \alpha_{1}$ radiation.

The compositional homogeneity of samples was shown by plots of permittivity $\left(\varepsilon^{\prime}\right)$ against temperature, Fig. 1 .

TABLE I. Lattice parameters, pellet densities, $\mathrm{T}_{\mathrm{c}}, \mathrm{T}_{0}$, and $\mathrm{C}_{\mathrm{W}}$.

\begin{tabular}{|c|c|c|c|c|c|c|c|}
\hline \multirow[b]{2}{*}{ Composition } & \multirow[b]{2}{*}{ Reference } & \multicolumn{2}{|c|}{$\begin{array}{l}\text { Lattice parameters } \\
(\AA)\end{array}$} & \multirow{2}{*}{$\begin{array}{l}\text { Density } \\
(\%)\end{array}$} & \multirow{2}{*}{$\begin{array}{c}\mathrm{T}_{\mathrm{c}} \\
\left({ }^{\circ} \mathrm{C}\right)\end{array}$} & \multirow{2}{*}{$\begin{array}{c}\mathrm{T}_{0} \\
\left({ }^{\circ} \mathrm{C}\right)\end{array}$} & \multirow{2}{*}{$\begin{array}{l}\mathrm{C}_{\mathrm{W}} \\
\left({ }^{\circ} \mathrm{C}\right)\end{array}$} \\
\hline & & $a$ & $c$ & & & & \\
\hline $\mathrm{BaTiO}_{3}$ & BT & $3.9953(2)$ & $4.0313(3)$ & 87 & 127 & 120 & 108000 \\
\hline $\mathrm{Ba}_{0.99} \mathrm{Ca}_{0.01} \mathrm{TiO}_{3}$ & BCT1 & $3.9912(3)$ & $4.0336(5)$ & 90 & 130 & 124 & 104000 \\
\hline $\mathrm{Ba}_{0.97} \mathrm{Ca}_{0.03} \mathrm{TiO}_{3}$ & ВСТ3 & $3.9895(4)$ & $4.0329(7)$ & 90 & 129 & 123 & 95000 \\
\hline $\mathrm{BaTi}_{0.99} \mathrm{Ca}_{0.01} \mathrm{O}_{2.99}$ & BTC1 & $3.9951(3)$ & $4.0313(4)$ & 91 & 107 & 97 & 130000 \\
\hline $\mathrm{BaTi}_{0.97} \mathrm{Ca}_{0.03} \mathrm{O}_{2.97}$ & BTC3 & $4.0080(4)$ & $4.0227(5)$ & 92 & 64 & 60 & 120000 \\
\hline
\end{tabular}

\footnotetext{
${ }^{a)}$ Electronic mail: a.r.west@sheffield.ac.uk.
} 


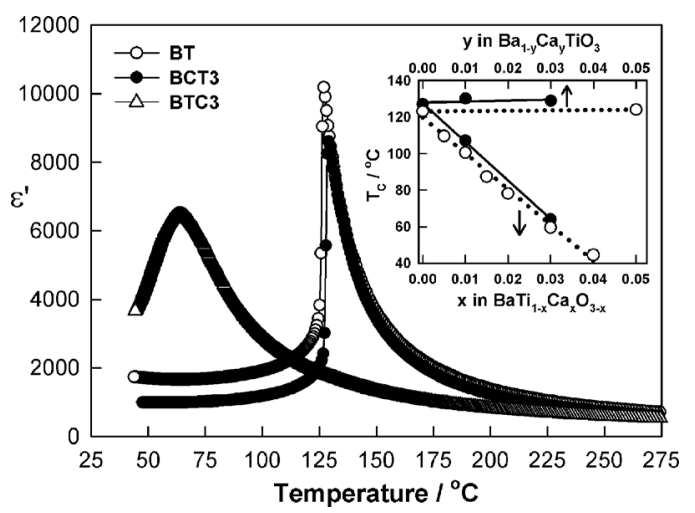

FIG. 1. Fixed frequency permittivity data at $100 \mathrm{kHz}$. Inset shows $\mathrm{T}_{\mathrm{c}} \mathrm{vs}$ composition with data from Ref. 3 (open symbols) for comparison. At 100 $\mathrm{kHz}$ the permittivity data represent the bulk capacitance, $\mathrm{C}_{1}$.

Single peaks in $\varepsilon^{\prime} / \mathrm{T}$ profiles were obtained; peak temperatures were largely independent of composition on A-site substitution but decreased significantly for B-site substitution. For samples that were inhomogeneous, the $\varepsilon^{\prime} / \mathrm{T}$ profiles showed doubled peaks indicating a distribution of $\mathrm{T}_{\mathrm{c}}$ values throughout the sample. This was particularly problematic for B-site doped samples fired at $1400{ }^{\circ} \mathrm{C}$ which required further heating at $1500{ }^{\circ} \mathrm{C}$ to achieve homogenization. The compositional homogeneity of fully fired samples contrasts with that typical of MLCCs which have compositional gradients associated with partial in-diffusion of dopants. The variation in $T_{c}$ with composition, inset to Fig. 1, is very similar to that reported in the literature. ${ }^{3}$

Impedance data were recorded over the range 150 to $600{ }^{\circ} \mathrm{C}$, both with and without an applied $d c$ bias of $10 \mathrm{~V}$ which, with pellet thicknesses of $\sim 1 \mathrm{~mm}$, corresponded to a potential gradient of $\sim 100 \mathrm{~V} \mathrm{~cm}^{-1}$. Impedance data formed two arcs from which resistance values $R_{1}$ and $R_{2}$ could be obtained from intercepts on the real $Z^{\prime}$ axis. From the magnitudes of the associated capacitance values, $C_{1}$ represented the bulk capacitance of the sample, as shown in Fig. 1. The capacitance of the lower frequency component $\mathrm{C}_{2}$ was in the range $0.4-4 \mathrm{nF} \mathrm{cm}^{-1}$.

Arrhenius plots of $\mathrm{R}_{1}^{-1}$ and $\mathrm{R}_{2}^{-1}$ are shown in Fig. 2. For BCT1 and BCT3, the data sets without $d c$ bias gave essentially linear plots with activation energies $0.62(1)$ to $0.81(1)$ $\mathrm{eV}$ for the bulk conductivity, $\mathrm{R}_{1}^{-1}$ and higher values, $0.78(1)$ to $1.36(1) \mathrm{eV}$, for $\mathrm{R}_{2}^{-1}$. For BTC1 and BTC3, $\mathrm{R}_{1}^{-1}$ and $\mathrm{R}_{2}^{-1}$ have similar activation energies but conductivity values are one to three orders of magnitude higher than for BCT1 and BCT3.

The effect of applying a $d c$ bias is very different in the two cases. For BCT samples, a small increase in conductivity for both components is seen and the bulk data show curved Arrhenius plots. For BTC samples, however, $\mathrm{R}_{1}^{-1}$ and $\mathrm{R}_{2}^{-1}$ increase by one to two orders of magnitude. The increases depend on both time and magnitude of the $d c$ bias and are fully reversible on removal of the $d c$ bias as shown for one example in Fig. 3. Measurements were carried out first in $\mathrm{N}_{2}$, second in air and finally in $\mathrm{O}_{2}$. As well as the conductivity increases with $d c$ bias, the conductivity increased with $\mathrm{P}_{\mathrm{O}_{2}}$ showing that, as a consequence of absorption of $\mathrm{O}_{2}$ at the sample surface and the following reaction:

$$
\mathrm{O}_{2}+4 \mathrm{e} \rightarrow 2 \mathrm{O}^{2-}
$$

the origin of the conductivity is $p$-type electronic. On appli-

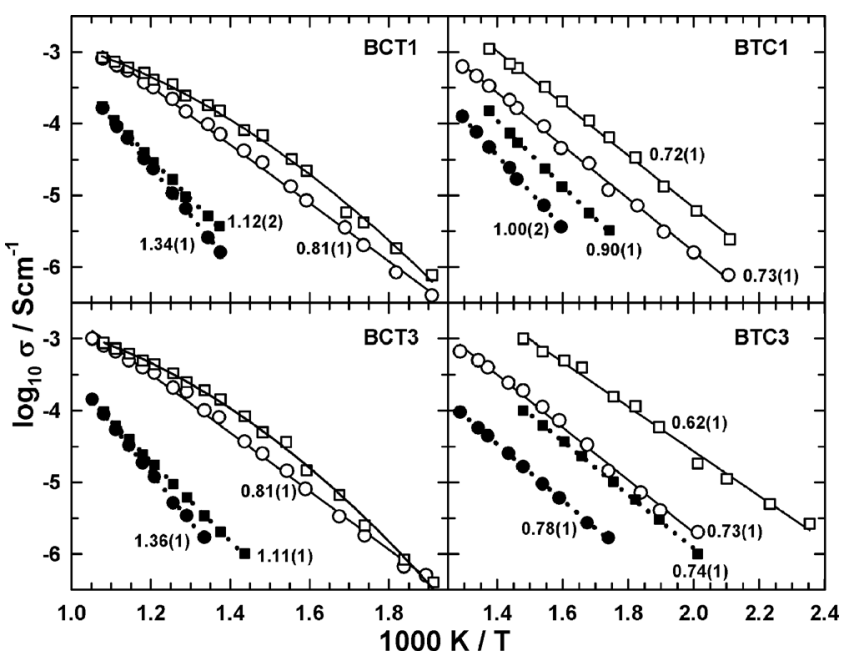

FIG. 2. Conductivity Arrhenius plots for $\mathrm{R}_{1}$ (open symbols) and $\mathrm{R}_{2}$ (filled symbols) measured without a $d c$ bias (circles) and with an applied voltage of $10 \mathrm{~V}$ (squares) after a steady state conductivity had been reached; sample thickness $1.44 \mathrm{~mm}$, BCT1; $1.52 \mathrm{~mm}$, BCT3; $1.19 \mathrm{~mm}$, BTC1; and $0.98 \mathrm{~mm}$, BTC3; activation energies in electron volt.

cation of a $10 \mathrm{~V} d c$ bias, the impedance in all three atmospheres showed that $\mathrm{R}_{1}^{-1}$ increased before leveling off after several hours. On removal of the $d c$ bias, the conductivity gradually returned to its original value. These experiments demonstrate that conduction is $p$-type electronic rather than by oxygen vacancies. In a separate experiment, the conductivity changes observed on application and removal of the $d c$ bias were similar when samples were maintained under vacuum for the duration of the experiment; this showed conclusively that oxygen exchange between sample and atmosphere was not involved.

We reported ${ }^{7,8}$ similar increases in conductivity with $d c$ bias in other acceptor-doped BT systems with B-site substitution by $\mathrm{Zn}^{2+}$ and $\mathrm{Mg}^{2+}$. No such effects are observed with stoichiometric BT, however a small effect is seen, here, with isovalent, Ba-site substitution of $\mathrm{Ca}$. Previous studies with donor-doped materials $\left(\mathrm{Nb}^{5+}\right.$ for $\mathrm{Ti}^{4+}$ and $\mathrm{La}^{3+}$ for $\left.\mathrm{Ba}^{2+}\right)$ did not show any $d c$ bias-dependence of the conductivity. The present results are therefore fully consistent with earlier studies; only samples that are acceptor-doped, with oxygen vacancies for charge compensation, show significant fieldenhanced bulk conductivity.

Impedance data show that voltage-dependent conductivities are observed in both grains and grain boundaries, Fig. 2, and are not dependent on the electrode material. ${ }^{7,8}$ They are not associated with electrochemical decomposition of the samples, nor with creation of compositional gradients associated with ionic migration and charge build-up. ${ }^{7,8}$ They are not associated with $\mathrm{O}_{2}$ exchange at the sample-atmosphere interface, nor with charge injection into the conduction band. ${ }^{7,8}$ The conducting species are electronic in spite of the widely-held view that oxygen vacancies are mobile in acceptor-doped materials that have oxygen vacancies for charge compensation. ${ }^{7,8}$ Further, the data represent longrange conductivities since there is no evidence of any blocking series capacitance in the conduction pathway in addition to the parallel resistance-capacitance (RC) elements that represent conduction through grains and grain boundaries; consequently, the conductivities do not represent localized hopping of dipoles such as $\mathrm{Ca}_{\mathrm{Ti}}^{\prime \prime}-\mathrm{V}_{\mathrm{O}}^{\bullet \bullet}$ pairs. 


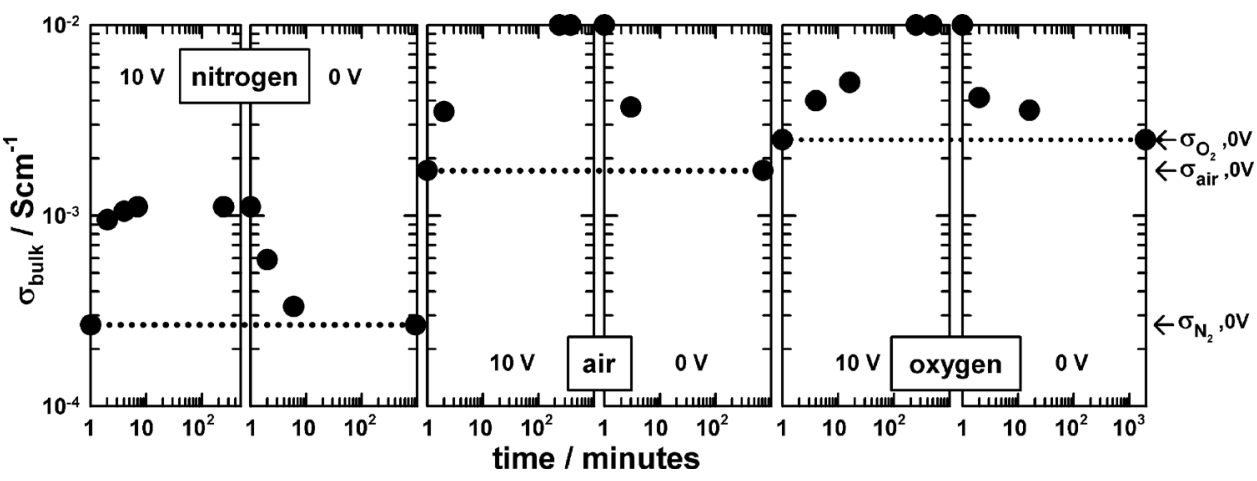

FIG. 3. Bulk conductivity at $600{ }^{\circ} \mathrm{C}$ for BTC1 after applying and removing a $d c$ bias of $10 \mathrm{~V}$ (sample thickness $1.19 \mathrm{~mm}$ ) at different times in $\mathrm{N}_{2}$, air, and $\mathrm{O}_{2}$.
A model to account for the voltage-dependent conductivity has been proposed ${ }^{7,8}$ which involves, first the creation of defect complexes associated with the acceptor dopant, i.e., $\mathrm{Zn}_{\mathrm{Ti}}^{\prime \prime}, \mathrm{Mg}_{\mathrm{Ti}}^{\prime \prime}$, and in the present case, $\mathrm{Ca}_{\mathrm{Ti}}^{\prime \prime}$. The second step is ionization of $\mathrm{O}^{2-}$ ions surrounding the positively charged acceptor sites. It is well-known that the second electron affinity of oxygen is positive; the $\mathrm{O}^{2-}$ ion, which is unstable in the gas phase, is stabilized in crystal lattices only by the large increase in lattice energy associated with $\mathrm{O}^{2-}$ ions compared with $\mathrm{O}^{-}$ions. An $\mathrm{O}^{2-}$ ion adjacent to an acceptor site does not have the same degree of stabilization as an oxide ion in a defect-free crystal lattice and is suggested to ionise readily in a relatively small potential gradient, leading to an overall increase in conductivity. Since the conduction mechanism is $p$-type, holes associated with the $\mathrm{O}^{-}$ions appear to be the principal current carriers, rather than electrons which, presumably, are trapped in Ti $3 d$ levels.

The results in Fig. 3 show that the level of $p$-type conductivity can increase by two methods, application of a $d c$ bias and increasing the oxygen partial pressure. The latter causes the filling of oxygen vacancies, primarily at the sample surface, with the anticipated following mechanism:

$$
\mathrm{V}_{\mathrm{O}}^{*}+1 / 2 \mathrm{O}_{2}+\mathrm{O}_{\mathrm{O}}^{\mathrm{x}} \rightarrow 2 \mathrm{O}_{\mathrm{O}}^{\bullet}
$$

in which an $\mathrm{O}^{2-}$ ion near the oxygen vacancy transfers an electron to the oxygen atom that enters the vacancy. Further work is required to establish how the field dependence changes with reducing oxygen partial pressure, as conductivity changes from $p$-type to electrolytic domain to $n$-type regions. It is also important to establish whether underbonded $\mathrm{O}^{2-}$ ions in other defect enviroments, e.g., at surfaces, are able to ionise.

Hopping conduction of either electrons or ions is usually unaffected by a small $d c$ bias; conduction in a particular direction occurs only because the $d c$ field applies a slight bias to the otherwise random hopping motion. In the present system, and for other acceptor-doped systems, ${ }^{7,8}$ impedance analysis using $\mathrm{M}^{\prime \prime} / \mathrm{Z}^{\prime \prime}$ spectroscopic plots showed that the more highly conducting state is achieved via a nucleation and growth process which is believed to commence at the sample-electrode interface at which the local potential gradient may be significantly greater than the average gradient across the entire sample of $\sim 100 \mathrm{~V} \mathrm{~cm}^{-1}$.

It is relevant to consider whether the voltage-dependent conductivity reported here is related to electrical degradation usually observed at much higher bias voltages. ${ }^{10}$ The enhanced conductivity at low fields reaches a steady state with increasing time, Fig. 3; at a given temperature, it also reaches a steady state with increasing field. ${ }^{7,8}$ It appears that low field-enhanced conductivity is not primarily responsible for degradation which is a runaway high field effect believed to be associated with migration of oxygen vacancies. Nevertheless, enhanced electronic conductivity may influence oxygen exchange kinetics at the sample-electrode interface and thereby, act as a precursor to degradation. Further studies are required on the mixed electronic/ionic conductivity and oxygen exchange kinetics as a function of $d c$ bias.

We thank EPSRC and "Bancaja-Universitat Jaume I" (Grant No. P1 1B2006-25) for support and Generalitat Valenciana for a fellowship BFPI/2007/174.

${ }^{1}$ Y. H. Han, J. B. Appleby, and D. M. Smyth, J. Am. Ceram. Soc. 70, 96 (1987).

${ }^{2}$ Y. Sakabe, Curr. Opin. Solid State Mater. Sci. 2, 584 (1997).

${ }^{3}$ L. Zhang, O. P. Thakur, A. Feteira, G. M. Keith, A. G. Mould, D. C. Sinclair, and A. R. West, Appl. Phys. Lett. 90, 142914 (2007).

${ }^{4}$ L. Zhang, L. Ben, O. P. Thakur, A. Feteira, A. G. Mould, D. C. Sinclair, and A. R. West, J. Am. Ceram. Soc. 91, 3101 (2008).

${ }^{5}$ P. V. Lambeck and G. H. Jonker, J. Phys. Chem. Solids 47, 453 (1986).

${ }^{6}$ H. Beltrán, E. Cordoncillo, P. Escribano, D. C. Sinclair, and A. R. West, J. Am. Ceram. Soc. 87, 2132 (2004).

${ }^{7}$ H. Beltrán, M. Prades, N. Masó, E. Cordoncillo, and A. R. West, J. Am. Ceram. Soc. 93, 500 (2010).

${ }^{8}$ M. Prades, N. Masó, H. Beltrán, E. Cordoncillo, and A. R. West, J. Mater. Chem. 20, 5335 (2010).

${ }^{9}$ J. R. Macdonald, in Impedance Spectroscopy: Emphasizing Solids Materials and Systems, edited by J. R. Macdonald (Wiley, New York, 1987), Chap. 2, pp. 27-132.

${ }^{10}$ S.-H. Yoon, C. A. Randall, and K.-H. Hur, J. Am. Ceram. Soc. 92, 1758 (2009); 92, 1766 (2009); 92, 2944 (2009). 